\title{
Noise Loading Analysis using Volterra Kernels to Characterize Fiber Nonlinearities
}

\author{
Jong-Hyung Lee ${ }^{\dagger}$ \\ Department of Electronic Engineering, Dongeui University, Busan 614-714, Korea
}

(Received October 29, 2012; Revised manuscript December 3, 2012; Accepted December 4, 2012)

\begin{abstract}
We derive analytical expressions for the output spectral density and the noise power $\mathrm{P}_{\beta}$ in noise loading analysis using Volterra kernels to characterize fiber nonlinearities. The bandwidth of the input noise source has little effect on $\mathrm{P}_{\beta}$, but the power of the input noise source and the dispersion parameter value of the fiber have a significant effect on $\mathrm{P}_{\beta}$. The Volterra method predicts $\Delta P_{\beta}[d B]=30 \mathrm{~dB} /$ decade, which agrees very accurately over a wide range of fiber parameters compared with the numerical results by the split-step Fourier method. Therefore the Volterra method could be useful to predict the performance of a dense WDM system when we plan to upgrade fiber or increase signal power.
\end{abstract}

Keywords: Fiber nonlinearities, Noise loading analysis, Volterra kernel, Split-step Fourier

OCIS codes: (060.2270) Fiber characterization; (060.0060) Fiber optics and optical communications; (060.4510) Optical communications

\section{INTRODUCTION}

One of the major concerns in multi-channel communication systems is the system impairment due to cross-talk between channels. Cross-talk is often induced by system nonlinearities because nonlinearities can generate new spectral components which may fall into the neighboring channels. In a dense wavelength division multiplexing(DWDM) system where channels are spaced very closely, the broadening effects on the spectrum due to various nonlinear effects like self-phase modulation(SPM), cross-phase modulation(CPM), and four-wave mixing(FWM) are in practice indistinguishable. In such a system, noise loading analysis could be useful in assessing the effects of broadened spectrum due to fiber nonlinearities on system performance.

In the noise loading analysis, a sharp notch filter is used to remove a part of the noise spectrum before input to the system. At the output of the system, a bandpass filter(BPF) tuned to the notch filter will indicate the spectral shape due to nonlinearities within the filter bandwidth. Therefore, the output of the BPF is the quantity of interest in the noise loading analysis. Some papers have discussed the noise loading analysis when the nonlinearities are modeled as memoryless [1-3]. Maqusi applied the Volterra series representation of nonlinearities in the noise loading analysis to model memory in nonlinear systems ${ }^{[4]}$. (Unfortunately, 'noise loading' has been used for a different meaning in optical communication systems. In the references ${ }^{[5,6]}$, noise loading means adding noise at the receiver in a controlled way to estimate bit-error rate(BER) margin.) Lee applied the Volterra kernel method in the noise loading analysis for the first time to assess fiber nonlinearities in optical communication systems assuming the fiber is lossless ${ }^{[7]}$.

In this paper, we derived $\Delta P_{\beta}[d B]$ from the Volterra kernel method in the noise loading analysis including the fiber loss, and the applicable range of the method is specified by comparing with the split-step Fourier(SSF) method as a reference.

\section{NOISE LOADING ANALYSIS USING VOLTERRA KERNEL}

The test setup for the noise loading analysis is quite simple as depicted in Fig. 1. The test consists of a broadband noise source followed by a notch filter. The bandwidth $\left(\mathrm{B}_{\mathrm{O}}\right)$ rejected

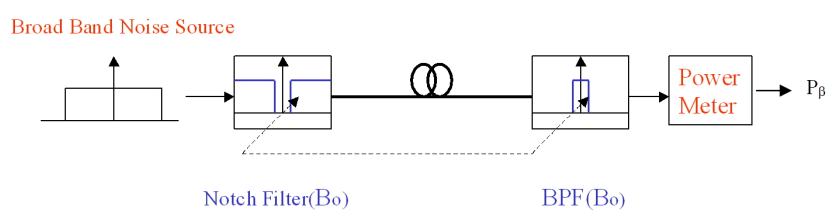

FIG. 1. Test set-up for the noise loading analysis in a fiber optic communication system

\footnotetext{
${ }^{\dagger}$ E-mail: jonghlee@deu.ac.kr

Color versions of one or more of the figures in this paper are available online.
} 


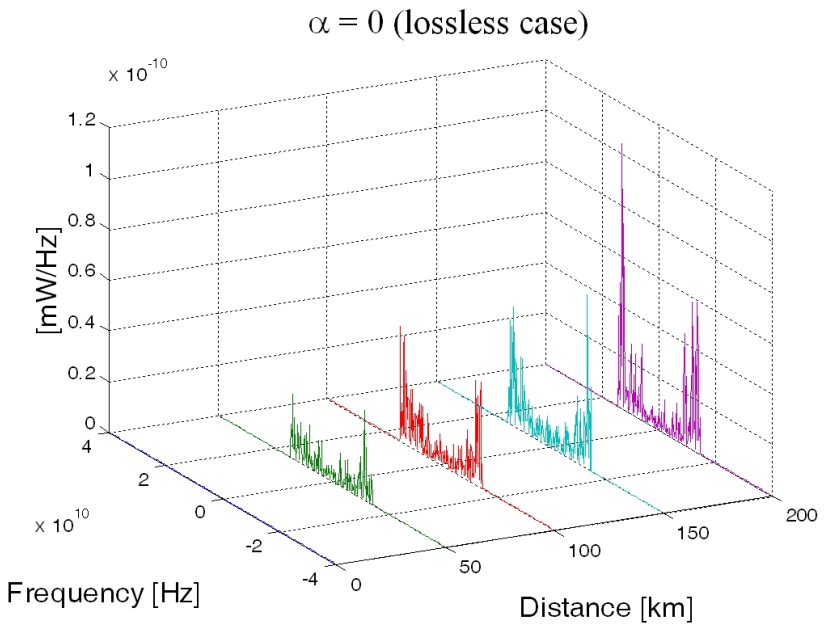

FIG. 2. Spectral growth within the notch filter bandwidth when $\beta_{2}=3\left[\mathrm{ps}^{2} / \mathrm{km}\right]$ and $\alpha=0$.

by the notch filter is designed to be much narrower than the bandwidth of the flat source. At the output of the fiber under test, an optical BPF, the bandwidth and center frequency of which is tuned to the notch filter, is inserted. Ideally, the output of the BPF corresponds to intermodulation noise due to fiber nonlinearities that falls into the bandwidth defined by the notch filter.

For example, Fig. 2 shows the growth with distance of the spectral densities which fall within the bandwidth of the output BPF. The split-step Fourier method is used to simulate the configuration depicted in Fig. 1. The fiber is modeled to have the second order propagation constant $\beta_{2}=3\left[\mathrm{ps}^{2} / \mathrm{km}\right]$, the fiber nonlinear coefficient $\gamma=2.6 \times 10^{-3}\left[\mathrm{~mW}^{-1} \mathrm{~km}^{-1}\right]$, while the fiber loss is ignored $(\alpha=0)$ to emphasize the effect of fiber nonlinearities.

While the simulation setup for the noise loading analysis is relatively simple, it may not be easy to deal with the simulation results because major quantities of concern have a stochastic nature. Therefore it is of interest to have an analytical expression for the noise loading analysis.

When fiber nonlinearities are weak, we may treat the solution of the nonlinear Schrödinger equation as a perturbation of the linear solution ${ }^{[8]}$ or equivalently we may express the output of the fiber with Volterra series transfer functions ${ }^{[9,10]}$. If we take only the first higher order Volterra series, the output of a single-mode fiber may be modeled as below.

The linear transfer function (the first order Volterra kernel), $H_{1}(\omega, z)=\exp \left(-\frac{\alpha}{2} z\right) \exp \left(j \frac{\beta_{2}}{2} \omega^{2} z\right)$, is derived from the nonlinear Schrödinger equation by assuming dispersion alone, and the third-order Volterra kernel(transfer function), $H_{3}$, may be expressed as below ${ }^{[9]}$.

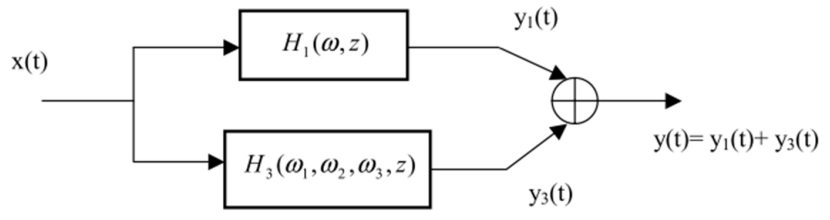

FIG. 3. Modeling of single-mode fibers with Volterra kernels.

$$
H_{3}\left(\omega_{1}, \omega_{2}, \omega_{3}, z\right)=j \gamma e^{-\frac{\alpha}{2} z+\frac{j}{2} \beta_{2}\left(\omega_{1}-\omega_{2}+\omega_{3}\right)^{2} z} \frac{e^{-\alpha z+j \beta_{2}\left(\omega_{1}-\omega_{2}\right)\left(\omega_{3}-\omega_{2}\right) z}-1}{-\alpha+j \beta_{2}\left(\omega_{1}-\omega_{2}\right)\left(\omega_{3}-\omega_{2}\right)},
$$

From Fig. 3, the output auto-correlation function, $R_{y y}(\tau)$, is obtained as below. $\mathrm{x}(\mathrm{t})$ and $\mathrm{y}(\mathrm{t})$ denote the input process to the fiber and the output process of the fiber, respectively.

$$
R_{y y}(\tau)=E\left\{y^{*}(t) y(t+\tau)\right\}=R_{y 1 y 1}(\tau)+R_{y 1 y 3}(\tau)+R_{y 1 y 3}^{*}(\tau)+R_{y 3 y 3}(\tau),
$$

where * denotes the complex conjugate and $E\{\cdot\}$ is the ensemble average operator. The output spectral density function, $S_{y y}(f)$, which is the Fourier transform of $R_{y y}(\tau)$, is then

$$
S_{y y}(f)=S_{y 1 y 1}(f)+S_{y 3 y 3}(f)+S_{y 1 y 3}(f)+S^{*}{ }_{y 1 y 3}(f),
$$

The spectral density functions for the third-order nonlinearity are derived in the literature ${ }^{[11]}$, and the results are

$$
\begin{aligned}
& S_{y 1 y 1}(f, z)=\left|H_{1}(f, z)\right|^{2} S_{x x}(f), \\
& S_{y 1 y 3}(f, z)=3 H_{1}^{*}(f, z) S_{x x}(f) \int H_{3}(u,-u, f, z) S_{x x}(u) d u, \\
& S_{y 3 y 3}(f, z)=9 S_{x x}(f)\left|\int H_{3}(u,-u, f, z) S_{x x}(u) d u\right|^{2} \\
& \quad+6 \iint\left|H_{3}(u, v-u, f-v, z)\right|^{2} S_{x x}(u) S_{x x}(v-u) S_{x x}(f-v) d u d v
\end{aligned}
$$

where $S_{x x}(f)$ is the input spectral density function after the notch filter in Fig. 1.

In the noise loading analysis, the average output power $\left(\mathrm{P}_{\beta}\right)$ of the BPF at the end of the fiber under test might be the most important quantity because $\mathrm{P}_{\beta}$ is the noise power due to fiber nonlinearities. In the evaluation of $\mathrm{P}_{\beta}$, the input spectral density $S_{x x}(f)$ has a sharply notched-out spectral shape. If the notch filter is placed at the center frequency of the input noise source, then $S_{x x}(f)=0$ from $-\mathrm{B}_{0} / 2$ to $+\mathrm{B}_{0} / 2$. Therefore, from Eq.(3) to (6), we observe that the second term of Eq.(6) is the only term that can contribute to $\mathrm{P}_{\beta}$. 
If we denote the second term of Eq. (6) as $S_{\beta}(f, z), \mathrm{P}_{\beta}$ can be evaluated by integrating $S_{\beta}(f, z)$ from $-\mathrm{B}_{\mathrm{o}} / 2$ to $+\mathrm{B}_{\mathrm{o}} / 2$. That is

$$
P_{\beta}=\int_{-B_{o} / 2}^{B_{o} / 2} S_{y y}(f, z) d f=\int_{-B_{o} / 2}^{B_{o} / 2} S_{\beta}(f, z) d f,
$$

We can observe that $S_{\beta}(f, z)$ is an even function of $\beta_{2}$ because $\left|H_{3}\right|^{2}$ is an even function of $\beta_{2}$. Therefore the Volterra kernel approach predicts that $S_{\beta}(f, z)$ and $\mathrm{P}_{\beta}$ depend only on the magnitude of the dispersion parameter.

\section{COMPARISON WITH SPLIT-STEP FOURIER METHOD}

To compare analytical results by the Volterra kernel method (Eq. (7)) with numerical results by the split-step Fourier method, the fiber is assumed to be $50 \mathrm{~km}$ long and to have $\alpha$ $=0.2 \mathrm{~dB} / \mathrm{km}, \gamma=2.6 \times 10^{-3}\left[\mathrm{~mW}^{-1} \mathrm{~km}^{-1}\right], \mathrm{D}= \pm 4[\mathrm{ps} / \mathrm{nm} \cdot \mathrm{km}]$, which is the novel optical characteristics of, such as, NZ_DSF(Non-Zero Dispersion Shifted Fiber). While the notch filter bandwidth $\mathrm{B}_{\mathrm{O}}$ is fixed at $25 \mathrm{GHz}$, the noise source bandwidth in the evaluation of $\mathrm{P}_{\beta}$ is increased from $150 \mathrm{GHz}$ to $550 \mathrm{GHz}$. If we assume that the broadband noise source models multiple channels in a WDM system with $50 \mathrm{GHz}$ channel spacing, then the noise source bandwidth from 150 $\mathrm{GHz}$ to $550 \mathrm{GHz}$ corresponds to channel numbers from $\mathrm{N}_{\mathrm{ch}}=$ 3 to $\mathrm{N}_{\mathrm{ch}}=11$. The optical power of the noise source is now determined by the product of channel number $\mathrm{N}_{\mathrm{ch}}$ and the optical power per channel, $\mathrm{P}_{\mathrm{ch}}[\mathrm{mW}]$.

Because the split-step Fourier method gives a little different value at every simulation due to its stochastic nature, we simulate 20 times for each simulation parameter set in all the cases in this paper and show the mean values with the value ranges. From Fig. 4, we can also observe that $\mathrm{P}_{\beta}$ is a strong function of the signal power while relatively insensitive to the noise source bandwidth when $\mathrm{N}_{\mathrm{ch}}>4\left(\mathrm{~B}_{\mathrm{sig}}>200 \mathrm{GHz}\right)$.

Figure 4 also shows that the Volterra kernel method(solid line) using up to the third order kernel overestimates the noise power output, $\mathrm{P}_{\beta}$, compared to the SSF method(dashed line). This is because the Volterra method simplifies the interplay between dispersion and nonlinearities of the fiber as depicted in Fig. 3. While the third order kernel (Eq.(1)) models the interplay in the Volterra method regardless of fiber parameters, the SSF method calculates the interplay within a very short segment of the fiber successively so that the phase shift due to each segment of the fiber nonlinearity is below a

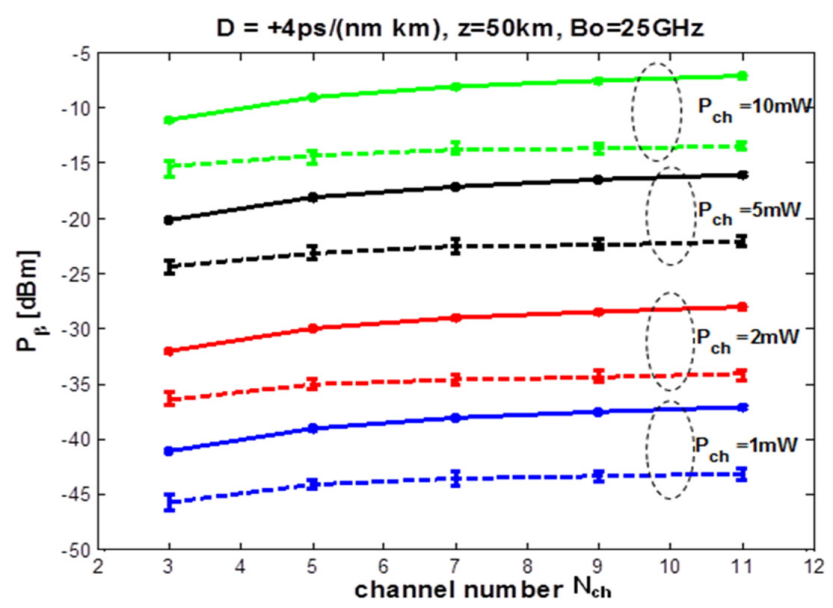

FIG. 4. Comparison of the calculated $\mathrm{P}_{\beta}$ when $\mathrm{D}=4 \mathrm{ps} /[\mathrm{nm}$. $\mathrm{km}$ ] (solid line: Volterra method, dashed line: SSF method).

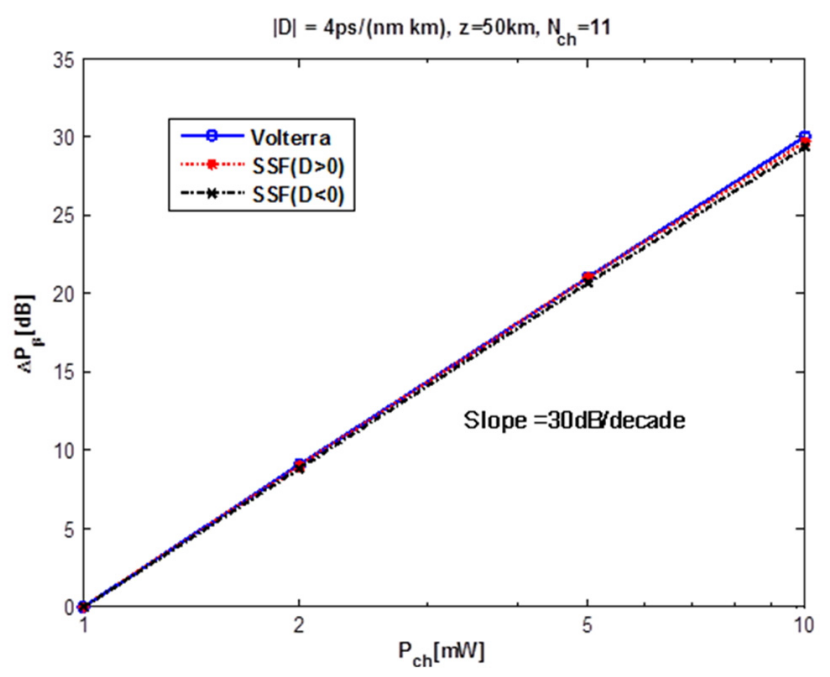

FIG. 5. $\Delta P_{\beta}[d B]$ as a function of $\mathrm{P}_{\mathrm{ch}}$ when $|\mathrm{D}|=4 \mathrm{ps} /[\mathrm{nm} \cdot \mathrm{km}]$.

certain small number, say 0.01rad.

Even though the Volterra kernel method using up to the third order kernel overestimates $\mathrm{P}_{\beta}$ compared to the SSF method, variations of $\mathrm{P}_{\beta}$ due to the different simulation parameters are quite consistent. Therefore the Volterra kernel method could be used to predict a relative value. The relative value of $\mathrm{P}_{\beta}$ can be defined as below.

$$
\Delta P_{\beta}[d B]=P_{\beta}(\text { new })[d B m]-P_{\beta}(\text { reference })[d B m],
$$

where $\mathrm{P}_{\beta}$ (reference) $[\mathrm{dBm}]$ is a reference value and $\mathrm{P}_{\beta}(\mathrm{new})[\mathrm{dBm}]$ is a new value obtained by a different set of simulation parameters.

If we take $\mathrm{P}_{\beta}$ when $\mathrm{P}_{\mathrm{ch}}=1[\mathrm{~mW}]$ as a reference, we can obtain Fig. 5 which shows $\Delta P_{\beta}[d B]$ as a function of $\mathrm{P}_{\text {ch. }}$. $\Delta P_{\beta}[d B]$ by the Volterra kernel method is quite predictable. 


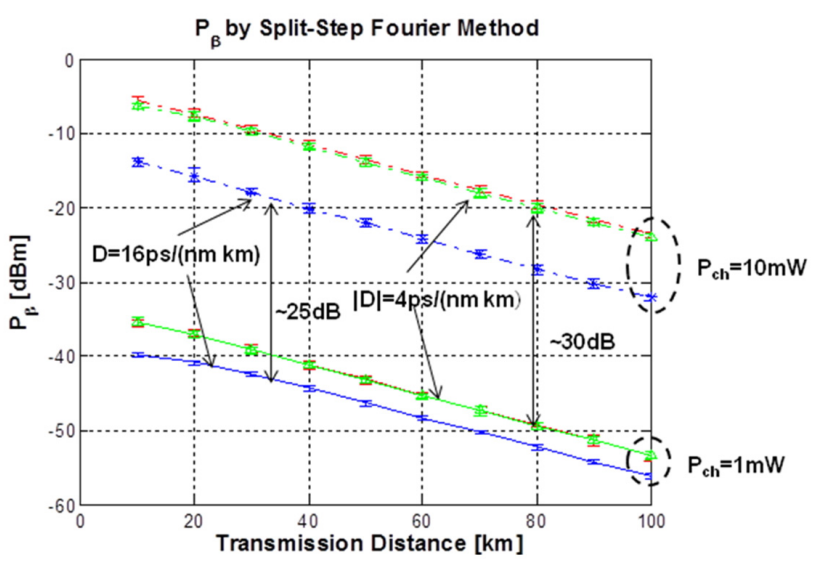

FIG. 6. $\mathrm{P}_{\beta}$ by SSF method when the transmission distance is varied (blue line: $\mathrm{D}=16 \mathrm{ps} /[\mathrm{nm} \cdot \mathrm{km}]$, red line: $\mathrm{D}=+4 \mathrm{ps} /[\mathrm{nm}$ $\cdot \mathrm{km}]$, green line: $\mathrm{D}=-4 \mathrm{ps} /[\mathrm{nm} \cdot \mathrm{km}])$.

If $\mathrm{P}_{c h}$ is increased twice compared to the reference value at a given transmission distance, the magnitude of the input spectral density $S_{x x}(f)$ will also be increased twice within $\mathrm{B}_{\mathrm{O}}$. Therefore from Eq.(7), if $\mathrm{P}_{\mathrm{ch}}$ is increased ten times, $\Delta P_{\beta}$ is predicted to increase $10 \log _{10}\left(10^{3}\right)=30[d B]$ regardless of the dispersion parameter, which agrees excellently with results shown in Fig 5 . When $|\mathrm{D}|=4 \mathrm{ps} /(\mathrm{nm} \cdot \mathrm{km}), \Delta P_{\beta}[d B] \mathrm{s}$ by the two methods, Volterra kernel method and SSF method, agree very closely (slope $\cong 30[\mathrm{~dB}] /$ decade) regardless of the dispersion region, $\operatorname{normal}(\mathrm{D}<0)$ or anomalous $(\mathrm{D}>0)$. Since the channel number $\left(\mathrm{N}_{\mathrm{ch}}\right)$ or equivalently the noise source bandwidth, affects very little on $\Delta P_{\beta}[d B]$, we only show $\Delta P_{\beta}[d B]$ when $\mathrm{N}_{\mathrm{ch}}=11$ in Fig. 5.

Figure 6 shows $P_{\beta}[d B m]$ by the SSF method when $\mathrm{N}_{\mathrm{ch}}=$ 10 while the transmission distance is varied. When $\mathrm{P}_{c h}$ is increased 10 times from $1 \mathrm{~mW}$ to $10 \mathrm{~mW},|\mathrm{D}|=4 \mathrm{ps} /(\mathrm{nm} \cdot \mathrm{km})$ result in $\Delta P_{\beta}[d B]=10 \log _{10}\left(10^{3}\right)=30[d B]$ regardless of the transmission distance, which is predicted by the Volterra method. However, when D $=16 \mathrm{ps} /(\mathrm{nm} \cdot \mathrm{km})$, the SSF method shows that $\Delta P_{\beta}[d B]$ is reduced to around $25 \mathrm{~dB}$.

Figure 7 shows $P_{\beta}[d B m]$ when $\mathrm{D}$ is varied. While $\Delta P_{\beta}[d B]$ by the Volterra kernel method is $30[\mathrm{~dB}]$ regardless of the dispersion parameter because the input power is increased ten times, $\Delta P_{\beta}[d B]$ by the split-step Fourier method is reduced to around $24[\mathrm{~dB}]$ when $\mathrm{D}$ is increased to $16 \mathrm{ps} /(\mathrm{nm} \cdot \mathrm{km})$, which agrees with results of Fig. 6. This is because the Volterra kernel method oversimplifies the interplay between dispersion and nonlinearities of the fiber by Eq.(1), the third order kernel. From Figs. 6 and 7, the Volterra kernel method may not be used to predict $\Delta P_{\beta}[d B]$ when the fiber is a conventional standard single-mode fiber $(\mathrm{D}=16 \sim 18 \mathrm{ps} /(\mathrm{nm}$. $\mathrm{km})$ ).

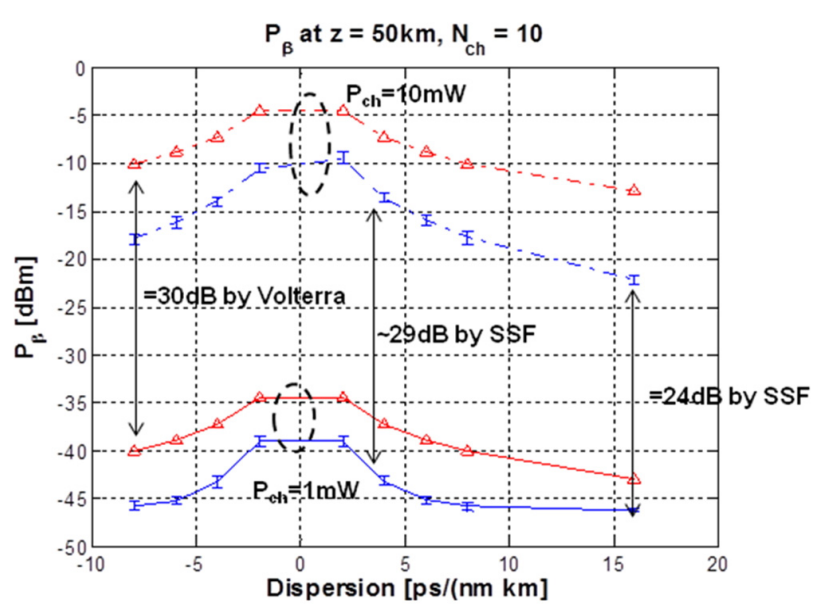

FIG. 7. $\mathrm{P}_{\beta}$ when the dispersion parameter is varied. ( $\Delta$ : Volterra method, I: SSF method).

\section{CONCLUSIONS}

We used the Volterra kernels for the first time to derive analytical expressions for the output noise spectral density $S_{\beta}(f, z)$ and the output noise power $\mathrm{P}_{\beta}$ in the noise loading analysis. The derived analytical expression is compared to the numerical SSF method. The Volterra kernel method using up to the third order kernel overestimates $\mathrm{P}_{\beta}$ in the noise loading analysis compared to the SSF method. Including higher order kernels could give us more accurate results. However, the Volterra kernel method with up to the third order kernel requires only moderate computational resources, while the method predicts $\Delta P_{\beta}[d B]$ very accurately over wide range of fiber parameters.

The bandwidth of the input noise source has little effect on $\mathrm{P}_{\beta}$, but the power of the input noise source and the dispersion parameter value affect significantly on $\mathrm{P}_{\beta}$. The Volterra kernel method predicts $\Delta P_{\beta}[d B]=30 \mathrm{~dB} /$ decade (or $9 \mathrm{~dB} /$ octave) when we vary the power of the input noise source. Simulation results by the SSF method agree well except when the fiber is a conventional standard single-mode fiber. In addition, unlike the SSF method, the Volterra kernel method does not have statistical variations in the noise loading analysis.

\section{ACKNOWLEDGMENT}

This work was supported by Dong-eui University Grant 2010AA168.

\section{References}

1. Y. L. Kuo, "Noise loading analysis of a memoryless 
nonlinearity characterized by a Taylor series of finite order," IEEE Transactions on Instrumentation and Measurement IM-22, 246-249 (1973).

2. G. C. Reis, "Further results in the noise loading analysis of a memoryless nonlinearity characterized by a Taylor series of finite order," IEEE Transactions on Instrumentation and Measurement IM-25, 28-33 (1976).

3. R. J. Fenton, "Noise-loading analysis for a general memoryless system," IEEE Transactions on Instrumentation and Measurement IM-26, 61-64 (1977).

4. M. Maqusi, "Noise loading analysis in FDM systems," IEEE Transactions on Communications COM-33, 1319-1323 (1985).

5. D. A. Fishman and B. S. Jackson, Optical Fiber Telecommunications IIIB (Academic Press, San Diego, USA, 1997), Chapter 3.

6. E. A. Golovchenko, V. J. Mazurczyk, and S. M. Abbott, "Implementing optical noise loading to estimate margin in
WDM systems," in Proc. OFC 1999 (San Diego, CA, USA, 1999), pp. 335-337.

7. J. H. Lee, Ph. D. Thesis, Virginia Tech, Blacksburg (2000), Chapter 5.

8. J. H. Lee, D. H. Han, and B. Y. Choi, "Analysis of fiber nonlinearities by perturbation method," J. Opt. Soc. Korea 9, 6-12 (2005).

9. K. V. Peddanarappagari and M. Brandt-Pearce, "Volterra series transfer function of single-mode fibers," J. Lightwave Technol. 15, 2232-2241 (1997).

10. K. V. Peddanarappagari and M. Brandt-Pearce, "Volterra series approach for optimizing fiber-optic communications system designs," J. Lightwave Technol. 16, 2046-2055 (1998).

11. J. S. Bendat, Nonlinear System Analysis and Identification from Random Data (John Wiley \& Sons, New York, USA, 1990), Chapter 4. 